\section{Safety of Drugs}

The Committee on Safety of Drugs, commonly known as the Dunlop Committee, has consolidated its place in the medical establishment in Britain in a relatively short time. Its second annual report, ${ }^{1}$ published this month, makes the point that experience has enabled the committee and the manufacturers making submissions to establish a good working arrangement. Indeed the fears of delay and obstruction that were expressed at the time of the creation of the committee have proved to be groundless.

In 1965 the committee considered 1,041 submissions in respect of new drugs. In 807 cases the committee had no objection to the proposals for marketing the drug or to clinical trials of it ; in 19 cases the committee could not agree to the proposals. Ninety-six submissions had been referred back to the manufacturers or were still being considered at the end of the year, and 119 had been withdrawn or were regarded as having been withdrawn because there had been no response to the committee's requests for further evidence. Surprisingly, the right of the committee to require evidence of the efficacy of a drug has been challenged by manufacturers on some occasions. In its report the committee makes the point that such evidence is essential, both because of the risks of prescribing an ineffective drug to a patient who is suffering from a serious illness for which satisfactory treatment exists, and because treatment of even trivial illness by a drug of unproved efficacy is not justified if such treatment carries the slightest risk to the patient.

The report also emphasizes that manufacturers should apply for approved names for new drugs at an early stage, and indeed the committee wishes to recommend that an approved name should be obtained before the publication of any clinical paper referring to a drug.

The committee states that it considers its responsibility for the safety of drugs is not limited to consideration of new drugs and adverse reactions. On the subject of the labelling of prescribed medicines the report states:

"The Committee believe that it would conduce greatly to the safe use of drugs if containers of prescribed medicines were labelled with the name of the medicine unless otherwise specified by the doctor. . . . The Committee therefore believe that the convention should be changed to require pharmacists to label prescribed medicines in the absence of a specific direction from the doctor to do otherwise. They believe that any suggestion that patients should be kept in ignorance of their treatment is quite inconsistent with contemporary medical thought. They realize however that there are occasions when in the patient's interest it may be undesirable for him to know what drug he is being given, but if this is the case a doctor should indicate this clearly to the pharmacist when he wrote his prescription."

A motion instructing the Council of the B.M.A. to press for this change was passed by a substantial majority at the Annual Representative Meeting earlier this month. ${ }^{2}$

The first complete year of operation of the early warning system was 1963. During the year 4,000 reports of suspected adverse reactions to drugs were submitted, most them coming from general practitioners. The field force of part-time medical officers followed up these reports whenever further action seemed indicated, and the committee comments that the doctors sending in the warning reports gave these field workers a most cordial reception. Some 200 of the larger

\footnotetext{
'Annual Roport of the Committee on Safety of Drugs, 1966. H.M.S.O., London.

2 Brit. med. F. Suppl., 1966, 2, 82.
}

hospitals are now taking part in a monitoring scheme by which each hospital sends monthly returns to the committee listing patients who have received certain drugs selected for report from that hospital. Adequate national coverage of all drugs on the list of specially monitored drugs has been achieved, and it has been possible to carry out surveys of the incidence in hospital of adverse reactions to any drug on that list.

Doctors and dentists have been informed of adverse reactions to drugs by a variety of methods. During the year a number of manufacturers have voluntarily agreed to warn doctors of adverse reactions to which the committee had drawn their attention, or have amended the literature on the drugs in question. In addition the committee has issued several warnings to the professions through the professional journals and has circularized a further paper in the "adverse reactions" series.

The report concludes by referring to proposed legislation to provide for many of the procedures which at present are carried out under voluntary arrangements with the pharmaceutical industry. The committee has not felt hampered by any lack of statutory powers. It is certainly important that any legislation should maintain the scope for flexibility and the exercise of professional responsibility which have been welcome features of the early years of drug control.

\section{Metabolism and Madness}

Attempts to find a biochemical basis for serious mental illnesses have a long history, going back into the last century It is a history full of disappointments, and many have doubted if chemistry would ever have anything useful to say about psychological disorders. Some observers have taken the extreme theoretical view that such disorders, being mental, could have only psychological origins and psychological treatment, as if mind could operate independently of brain. Others, conceding the importance of the physical, or regarding "mind" and "brain" as but two aspects of the same entity, have hoped to see an explanation of psychosis in terms of altered neural connexions or changed electrical properties in some part of the brain, awaiting discovery by neuropathologist or physiologist. This doubt about which is the correct scientific approach to mental disorder was expressed by an eminent biochemist who wrote, "It would take many biochemists a long time to find a noisy circuit in a radio receiver, if they restricted themselves to chemical techniques."1

However, hopes of a greater understanding through chemical discovery have received encouragement latterly from various sources. In British psychiatry, at least, this is the great era of successful physical treatment, whether by induced epileptic fits (usually electric convulsion treatment since 1937), or by chlorpromazine (since 1952), or imipramine (1957), or other chemical substances. This has suggested that metabolic processes in the brain-particularly those involving neurohormones such as noradrenaline, serotonin, or acetylcholine-may be important for its normal functioning and in psychological disorders. Endocrinology hints that mental functions alter in hypoglycaemia, in lack or excess of thyroid hormone, in adrenal corticosteroid disturbances, and under the influence of sex hormones. Clinical biochemistry itself has shown that mental subnormality may arise because 
of inherited defects in amino-acid metabolism; lack of sufficient nicotinamide or pyridoxine may precipitate mental disturbance or fits, and acute porphyria can be diagnosed as "hysteria." The significance of the last to our historical knowledge of Gcorge III was recently discovered by Ida Macalpine and R. Hunter. ${ }^{3}$ The accidental discovery of new chemicals which can cause hallucinations (lysergic acid diethylamide, phencyclidine, psilocybin) has renewed interest also in mescaline and in the chemical mechanisms of hallucinogenesis. ${ }^{4}$ The importance of the biochemical and physical approach both in the treatment of the mentally ill and in the provision of satisfactory medical education is emphasized by Dr. William Sargant in his Watson Smith lecture published in the B.M.F. this week (page 257).

It is true also tha $i$ the biochemist of today is better equipped than the biochemist of even twenty years ago. $\mathrm{He}$ has a wider repertoire of subtle techniques, he can draw on the flood of new discoveries in cell chemistry, tissue metabolism, and human medical biochemistry for his hypothesis, he is more aware of clinical difficulties, ${ }^{1}$ and above all his targets are more modest. Instead of setting out to discover the "metabolic error in schizophrenia" (which begs a good many questions straightaway, and is a very hit-or-miss programme of research) he is content to study any abnormality which shows up in psychiatric patients, whether it is the "cause" of the patient's illness or not. Thus abnormalities of magnesium metabolism have been found in alcoholism, of serum and urinary calcium in depression and some other disorders, ${ }^{5}$ and of renal control of sodium in some patients with cyclical psychoses. ${ }^{6}$ Such abnormalities require explanation, and the explanation is a permanent valid addition to the sum of human physiology, whether it helps psychiatrists or not.

In fact in the end it will help psychiatry. If over 70 years of psychiatric research have taught us anything it is that psychiatrists are faced with complex, difficult problems which they have been trying to solve on too narrow a basis with insufficient information. The human brain is an anatomically complex organ, its normal chemistry very obscure, its relationships with liver and endocrine glands ill understood. We are almost totally ignorant of the actions and fates of the drugs we use in treatment. We know hardly anything of the physical aspects of hunger or sleep or how body weight is regulated. We know virtually nothing about the laws controlling the behaviour of organisms with simpler nervous systems than our own-worms, spiders, insects, molluscs-by chemical means. And we do not know some of the simpler biochemical abnormalities detectable in psychiatric patients because we fail to look for them.

This is the importance of the recent work Dr. D. M. Shaw describes at page 262 . It does not alter the diagnosis or treatment of a single patient or explain depression in biochemical terms. But it is widening our metabolic knowledge of these patients; it is posing metabolic problems for solution; and out of this will eventually come some of the wider knowledge which will answer the question how much chemistry can do for psychiatry. At present Dr. Shaw and Dr. A. J. Coppen are almost alone in making these

\footnotetext{
1 Kety, S. S., Science, 1959, 129, 1528, 1590.

- Ackner, B., Cooper, J. E., Gray, C. H., and Kelly, M., Ұ. Psychosom. Res., 1962, 6, 1 .

s Macalpine, I., and Hunter, R., Brit. med. F., 1966, 1, 65.

- Ibid., 1966, 1, 1495.

Ibid., 1966, 1, 1495.

- Crammer, J. L., in Aspects of Psychiatric Research (p. 408), ed. D Richter, J. M. Tanner, Lord Taylor, and O. L. Zangwill, 1962. London.
}

studies at the clinical level, and there is plenty of scope for more research here. Alcoholism in its various aspectsaddiction to the alcohol itself, delirium tremens, hallucinosis, paranoia, dementia-ought to be an instructive field in which to study the interaction of biochemical and psychological factors in the production of disorder. For instance, there is the possibility that ethanol in customary doses disturbs transport of potassium. Arteriosclerotic dementia in the elderly, who so often have impaired renal, pulmonary, or cardiac function, runs a fluctuating course which suggests a metabolic factor at work, perhaps changes in blood $p H$ or electrolyte shifts. Manic-depressive patients with very frequent attacks may be almost continuously in hospital and offer opportunities for observation of abnormalities of electrolyte or aromatic aminoacid metabolism, and dietary control. Perhaps Dr. Shaw's paper will stimulate others to look beyond the daily routine, to take advantage of these opportunities, and build up some of the fundamental knowledge still lacking in psychological medicine.

\section{Acute Non-specific Pericarditis}

The fact that nineteen cases of acute non-specific pericarditis have been reported in this issue of the B.M.F. from one English district general hospital shows that this interesting condition is far from rare. The cases described by Dr. Anthony Martin (p. 279) are all typical examples of it, and, as usual, the patients all recovered, even though four presented with very severe chest pain and shock. The electrocardiographic changes in this form of pericarditis help to distinguish it from cardiac infarction, and other characteristic signs and symptoms were discussed in these columns last year. ${ }^{1}$ Nevertheless, many cases are probably still wrongly diagnosed as infarcts despite characteristic E.C.G. signs and the absence of enzyme changes. Some of these patients may be placed in danger of haemopericardium if they are given anticoagulants.

A keener awareness of the possibility of acute non-specific pericarditis should reveal many more cases and may in the end lead to a fuller understanding of its causation. Virus infection undoubtedly accounts for some cases, but probably for none in the present series. R. W. Strachan ${ }^{2}$ has listed eighteen conditions which may be associated with pericarditis, ranging from mumps to systemic lupus erythematosus, so it may well have a variety of causes, but a strong suspicion remains that there is a disease of distinct aetiology and that it may be an autoimmune condition. J. Robinson and W. W. Brigden $^{3}$ have shown that heart antibodies increase after mitral valvotomy, and that when they reach their peak the post-commissurotomy syndrome, which closely resembles acute non-specific pericarditis, will develop in some cases. The pericarditis which may follow a cardiac infarction, described by $\mathbb{W}$. Dressler ${ }^{4}$ and by C. Davidson, M. F. Oliver, and R. F. Robertson, ${ }^{5}$ probably belongs to the same group of disorders that may follow injury to the heart. Further research is clearly indicated.

\footnotetext{
Brit. med. F., 1965, 2, 60.

2 Strachan, R. W., Scot. med. F., 1963, 8, 402.

3 Robinson, J., and Brigden, W. W., Brit. med. F., 1963, 2, 706.

- Dressler, W., Arch. intern. Med., 1959, 103, 28.

Dressler, W., Arch. inter. med. 7., 1961. 2, 535.

6 Liu, H. Y., and Garcia, R., Amer. Heart 7., 1965, 69, 677

Krook, H., Acta med. scand., 1954, 148, 201.
} 